\section{Clinical Profile of Scrub Typhus in Newborns}

Scrub typhus can affect all age groups but has been rarely reported among neonates. We retrieved records of neonates admitted with scrub typhus from our hospital database, and analyzed their clinical and laboratory features. Only patients who were confirmed as having scrub typhus according to WHO criteria were included [1].

During the period from January, 2013 to December, 2018, a total of 525 patients were admitted with scrub typhus, out of which seven $(1.3 \%)$ were newborns. All the seven patients were from neighbouring districts of Chennai and had postnatal scrub typhus with fever, hepatosplenomegaly, thrombocytopenia and elevated C-reactive protein (CRP). Two cases had eschar (28\%). Affected babies had complications such as shock $(n=4)$, respiratory failure $(n=3)$, disseminated intra-vascular coagulation $(n=2)$ and multi organ dysfunction syndrome $(n=2)$. Other causes of fever were ruled out as dengue IgM, peripheral smear for malaria, TORCH (toxoplasmosis, rubella, cytomegalovirus, herpes, and others) screen and blood culture were negative.

Pediatric scrub typhus in a previous study [2] presented as prolonged fever $(100 \%)$, gastrointestinal symptoms (76\%), lymphadenopathy (96\%) and hepatosplenomegaly (61\%). Eschar was seen in $50(60 \%)$ patients and only six patients had severe illnesses (7\%) [2]. In our series, six babies who were treated with doxycycline ( 4.5 $\mathrm{mg} / \mathrm{kg} /$ day for 5 days) improved and there were no adverse reactions. One baby, treated with azithromycin $10 \mathrm{mg} / \mathrm{kg} /$ day died. Long-term follow-up data of the babies who survived was not available.

On review of English literature from 1992 to 2018 using Pub Med, 12 newborns with scrub typhus from seven publications [3-9] were identified, and nine (75\%) had postnatal scrub typhus (three were due to vertical transmission). Fever, hepatosplenomegaly and thrombocytopenia were present in all of them and only one had an eschar (8\%). Most common complications encountered were shock $(n=8)$, respiratory failure $(n=5), \mathrm{DIC}(n=4)$ and $\operatorname{MODS}(n=3)$. Four babies who were treated with doxycycline and two out of the four babies treated with azithromycin improved. The mortality rate was $25 \%$.
Scrub typhus should be considered in the differential diagnosis of newborns with fever, hepatosplenomegaly, thrombocytopenia and elevated CRP, especially in endemic regions. We found doxycycline to be useful in neonates without any side-effects.

Acknowledgements: Dr Arasar Seeralar, Dr Mangala Bharathi, Dr Arun Karthik, Dr Anitha, Ramya, Dr Devasena, Boopathi, Dr Shivashankar, Dr Ajay Kumar, Dr Avinash Kumar, Dr Divya Durga, Dr Manikandan, Nalini, Dr Padmesh, Dr Shanmuga Suntharam, Dr Shanmuga Priya and Dr Subasree for their support.

Contributors: TS: study concept, data collection and analyis, and manuscript preparation; CK: study concept and manuscript preparation.

Funding: None; Competing interest: None stated.

TE ABdul SAMAD AND CN KAMALARATHNAM*
Department of Neonatology,
Institute of Child Health and Hospital for Children,
Madras Medical College,
Chennai, India.
*drkamal.rathnam@gmail.com

\section{REFERENCES}

1. World Health Organization. WHO Recommended Surveillance Standards. Second edition. 2006. Available from: http://www. who.int/emc. Accessed September 15, 2019.

2. Lakshmanan S, Sagayaraj M, Sujatha B, Vasudevan $L D$. Clinical and laboratory profile of pediatric scrub typhus in a tertiary care teaching hospital in Southern India. Indian J Pediatr. 2018;5:2092-7.

3. Wang CL, Yang KD, Cheng SN, Chu ML. Neonatal scrub typhus: a case report. Pediatrics. 1992;89:965-8.

4. Suntharasaj T, Janjindamai W, Krisanapad S. Pregnancy with scrub typhus and vertical transmission: A case report. J Obstet Gynaecol. 1997;23:75-8.

5. Aarthi P, Bagyalakshmi R, Mohan KR, Krishna M, Nitin M, Madhavan HN, et al. First case series of emerging rickettsial neonatal sepsis identified by polymerase chain reaction-based deoxyribonucleic acid sequencing. Indian JMed Microbiol. 2013;31:343-348.

6. Gothwal S, Dhruw SS, Choudhry R, Sitaraman S. Neonatal scrub typhus. Pediatr Em Care Med Open Access. 2016;1:2.

7. Vajpayee S, Gupta RK, Gupta ML. Scrub typhus causing neonatal hepatitis with acute liver failure-A case series. Indian J Gastroenterol. 2017; 26:61-5.

8. Jajoo M, Kumar D, Manchanda S. Scrub typhus in a new born. J Clini Diag Res. 2017;11:05-06.

9. Paramanantham P, Sathyamoorthy P, Sekar P. Neonatal scrub typhus. World J Pharmaceutical Res. 2018;7; 836-40. 